\title{
Does Oxygen Transport Affect the Cell Voltages of Metal/Air Batteries?
}

\author{
Charles W. Monroe ${ }^{\mathrm{z}}$ \\ Department of Engineering Science, University of Oxford, Oxford OX1 3PJ, United Kingdom
}

\begin{abstract}
Simultaneous transport of an electrolyte and dissolved oxygen is analyzed with Newman's concentrated-solution theory to assess how nonuniform oxygen distributions might impact the voltages of metal/air batteries. For a solution comprising a neutral solvent, a simple salt, and oxygen, the Onsager-Stefan-Maxwell transport equations are inverted, yielding flux-explicit laws for oxygen, anion, and cation transport that distinguish the effects of individual diffusion and migration driving forces. Along with the ionic conductivity, electrolyte diffusivity, oxygen diffusivity, and cation transference number, a migration coefficient and a cross-diffusion coefficient are identified, which respectively account for the effects of electro-osmotic drag on oxygen and diffusional drag between salt and oxygen. A derived current/voltage relation reveals how oxygen gradients can in principle affect the cell potential; significant diffusion potential can arise from oxygen if it experiences electro-osmotic drag. Prior models are proven to follow from an assumption that cross-diffusion and electro-osmosis are both negligible, or, equivalently, that oxygen/ion interactions are weak. Experiments to quantify the novel transport properties are discussed, along with quantitative estimates of the cross diffusivity and migration coefficient.

(C) The Author(s) 2017. Published by ECS. This is an open access article distributed under the terms of the Creative Commons Attribution 4.0 License (CC BY, http://creativecommons.org/licenses/by/4.0/), which permits unrestricted reuse of the work in any medium, provided the original work is properly cited. [DOI: 10.1149/2.0521711jes] All rights reserved.

(cc) BY
\end{abstract}

Manuscript submitted April 3, 2017; revised manuscript received June 14, 2017. Published July 5, 2017. This paper is part of the JES Focus Issue on Mathematical Modeling of Electrochemical Systems at Multiple Scales in Honor of John Newman.

A standard liquid electrolyte for a metal/oxygen battery is made up of a neutral solvent, dissolved oxygen, and a salt with a single type of anion and a single type of cation. Given that four electrochemical species comprise these electrolytes, one expects on the basis of the Onsager mass-transport theory ${ }^{1}$ that six independent transport coefficients are needed to describe all the possible pairwise interactions associated with diffusion and migration. A recent paper by the author's group ${ }^{2}$ applies the Onsager-Stefan-Maxwell formalism to lithium/oxygen batteries, but assumes two of the six transport properties are negligible. Most other state-of-the-art continuum models of metal/oxygen-battery electrolytes incorporate only four transport coefficients. ${ }^{3-7}$ We set out here to address this issue by proposing convenient flux laws that account for dissipative interactions between oxygen flow and ion flow.

Macroscopic transport properties can yield information about microscopic interactions, and vice versa. By establishing relationships between the set of macroscopic transport coefficients and the StefanMaxwell diffusivities, one can determine what conditions must be met to reduce a complete transport model to the four-coefficient form used in most simulations.

Following Newman, ${ }^{8}$ flux-explicit transport equations can be derived from Onsager-Stefan-Maxwell constitutive laws by a sequence of linear transformations. A general implementation of Newman's approach, which also shows how to identify and count independent macroscopic transport properties for multicomponent electrochemical systems, was recently presented by Monroe and Delacourt. ${ }^{9}$ That inversion procedure yields a 'MacInnes equation' (extended form of Ohm's law ${ }^{8,10}$ ) expressing the current density $\vec{i}$ in terms of neutralspecies activity gradients, as well as the gradient of a single ionic electrochemical potential $\mu_{i}$ that can be related to the potential of a reference electrode. The portion of the electrode-potential drop arising from spatial salt-activity differences is commonly called 'diffusion potential' (or 'concentration overpotential'). Considering the complete set of proper mass-transport driving forces from irreversible thermodynamics suggests that diffusion potential might arise from differences in oxygen concentration, as well as from differences in salt concentration.

There is reason to believe that diffusion potentials from oxygen exist in some metal/oxygen batteries. For instance, experiments with

${ }^{\text {z} E-m a i l: ~ c h a r l e s . m o n r o e @ e n g . o x . a c . u k ~}$ aprotic $\mathrm{Li} / \mathrm{O}_{2}$ cells typically require a preconditioning interval, in which the system is held at open circuit while being exposed to oxygen at fixed pressure until the cell voltage equilibrates. ${ }^{11-14}$ During preconditioning, which can last for hours, ${ }^{12-14}$ the open-circuit potential can vary across tens or hundreds of millivolts. It is likely that some part of this variation arises from a diffusion potential: since salt flux is negligible and the interfacial kinetics of oxygen absorption into the liquid electrolyte is probably fast, relaxation of the oxygen distribution within the cell could be the source of the slow voltage change. This hypothesis is supported by the observation that far shorter preconditioning is needed when $\mathrm{Li} / \mathrm{O}_{2}$ cells are thinner. ${ }^{11}$

By inverting the Onsager-Stefan-Maxwell laws, both a MacInnes equation and flux laws in Nernst-Planck form (describing the excess fluxes of oxygen, cations, and anions relative to solvent explicitly, using terms that distinguish the impacts of individual diffusion and migration driving forces) can be derived. The flux-explicit laws naturally include six macroscopic transport properties bijectively related to the six Stefan-Maxwell coefficients.

Most existing models include four of the six properties that thermodynamic completeness requires: oxygen diffusivity, electrolyte diffusivity, ionic conductivity, and cation transference number. But the flux-law inversion process also suggests natural definitions for a 'migration coefficient', which determines how the flow of ionic current can drive oxygen flux, and for a 'cross-diffusion coefficient', which quantifies how salt gradients can drive oxygen flux (and how oxygen gradients can drive salt flux). The MacInnes equation for the fourspecies electrolyte further shows how oxygen migration may manifest a diffusion potential associated with oxygen gradients.

After the theory is developed, it is applied to analyze the preconditioning of planar lithium/oxygen cells. An estimate of the migration coefficient is provided, along with lower and upper bounds on the cross diffusivity. The analysis suggests that significant overpotential can arise from non-uniform oxygen distributions in $\mathrm{Li} / \mathrm{O}_{2}$ cells, and that oxygen flux due to migration could be comparable to the migratory fluxes of cations and anions.

\section{Macroscopic Transport Properties}

Monroe and Delacourt's procedure to develop thermodynamically complete transport laws in Nernst-Planck form ${ }^{9}$ begins with the arrangement of species indexes in an ordered list. (In this context, 'thermodynamically complete' means that the flux laws incorporate all 
the possible sources of energy dissipation identified from a local entropy balance.) Let indexes $\{0,0,-,+\}$ indicate the neutral-solvent, oxygen, anion, and cation species, respectively. Placement in the last position identifies cations as the sole charged species whose electrochemical potential will appear in the MacInnes equation that emerges from the Onsager-Stefan-Maxwell laws. This choice is natural here because cations are generally produced and consumed at the negative electrodes of metal/oxygen batteries, and reference electrodes reversible to cations are typically used.

Monroe and Delacourt's general treatment of electrochemical fluxlaw inversion can be applied to regions with space charge. ${ }^{9}$ For most applications, however, it is suitable to assume local electroneutrality, through the constraint

$$
F \sum_{i} z_{i} c_{i} \approx 0
$$

Here $F$ is Faraday's constant, and $z_{i}$ and $c_{i}$ respectively represent the equivalent charge and local molar concentration of species $i$. Since the stoichiometric coefficents $v_{i}$ in a salt formula unit relate through the Guggenheim condition $z_{+} \nu_{+}+z_{-} \nu_{-}=0$, electroneutrality allows one to write either of the ion concentrations in terms of an electrolyte concentration $c_{\mathrm{e}}: c_{\mathrm{e}}=c_{+} / \nu_{+}=c_{-} / \nu_{-}$. The total number of ions in a formula unit of salt will be written as $\nu_{\mathrm{e}}$ for brevity: $\nu_{\mathrm{e}}=\nu_{+}+\nu_{-}$.

Onsager-Stefan-Maxwell equations describing multicomponent transport in isothermal, isobaric phases are expressed in terms of electrochemical-potential-gradient diffusion driving forces as

$$
-c_{i} \vec{\nabla} \mu_{i}=\frac{R T}{c_{\mathrm{T}}} \sum_{j \neq i} \frac{c_{j} \vec{N}_{i}-c_{i} \vec{N}_{j}}{\mathscr{D}_{i j}},
$$

in which $R$ is the gas constant, $T$ the absolute temperature, $c_{\mathrm{T}}=$ $\sum_{i} c_{i}=c_{0}+c_{\mathrm{o}}+v_{\mathrm{e}} c_{\mathrm{e}}$ is the total molar concentration of particles in the solution, $\vec{N}_{i}$ is the molar flux of species $i$, and $\mathscr{D}_{i j}$ is the Stefan-Maxwell diffusivity of species $i$ through species $j$. Although four transport laws can be written in the form of Equation 2, only three are linearly independent because of the Gibbs-Duhem equation. The Onsager reciprocal relation for Stefan-Maxwell coefficients is a symmetry, $\mathscr{D}_{i j}=\mathscr{D}_{j i}$, so when applied to the four-species system here, Equations 2 only contain $4 \cdot(4-1) / 2=6$ distinct transport coefficients.

Since the oxygen and solvent species are uncharged, the StefanMaxwell diffusivity of oxygen through solvent, $\mathscr{D}_{\mathrm{o} 0}$, can be readily understood from a macroscopic point of view. (It can be approximated as equaling the thermodynamic diffusivity of oxygen through solvent, measured in the absence of salt.) When recasting Equations 2 in the Nernst-Planck form it will be convenient to exchange the other Stefan-Maxwell diffusivities for properties with similarly straightforward interpretations.

In Newman's standard concentrated-solution theory of binary electrolytes, ${ }^{8}$ the thermodynamic diffusion coefficient of electrolyte through solvent, $\mathscr{D}_{\mathrm{e} 0}$, and the cation transference number relative to solvent, $t_{+\phi}^{0}$, are defined in terms of Stefan-Maxwell diffusivities as

$$
\mathscr{D}_{\mathrm{e} 0}=\frac{\left(z_{+}-z_{-}\right) \mathscr{D}_{0+} \mathscr{D}_{0-}}{z_{+} \mathscr{D}_{0+}-z_{-} \mathscr{D}_{0-}} \quad \text { and } \quad t_{+\varnothing}^{0}=\frac{z_{+} \mathscr{D}_{0+}}{z_{+} \mathscr{D}_{0+}-z_{-} \mathscr{D}_{0-}} .
$$

It will be shown below that for an electrolytic solution which also contains dissolved oxygen, the definition of the cation transference number relative to solvent differs somewhat from the familiar form in Equation 3. Thus a subscript $\varnothing$ is added to $t_{+\varnothing}^{0}$ here, to emphasize that the symbol represents 'the cation transference number relative to solvent, as defined in the absence of oxygen.' The electrolyte conductivity as defined in the absence of oxygen, $\kappa_{\phi}$, is

$$
\frac{1}{\kappa_{\emptyset}}=-\frac{R T}{c_{\mathrm{T}} F^{2} z_{+} z_{-}}\left[\frac{1}{\mathscr{D}_{+-}}-\frac{z_{-} c_{0}}{\nu_{+} c_{\mathrm{e}}\left(z_{+} \mathscr{D}_{0+}-z_{-} \mathscr{D}_{0-}\right)}\right] .
$$

Values of $\mathscr{D}_{\mathrm{e} 0}, t_{+\phi}^{0}$, and $\kappa_{\phi}$ measured in oxygen-free liquid electrolytes can be used to estimate $\mathscr{D}_{0+}, \mathscr{D}_{0-}$, and $\mathscr{D}_{+-}$.
By analogy to $t_{+\varnothing}^{0}$ and $\mathscr{D}_{\mathrm{e} 0}$, it is helpful to identify a cation transference number relative to oxygen as defined in the absence of solvent, $t_{+\phi}^{\mathrm{o}}$, and a thermodynamic diffusivity of electrolyte through oxygen, $\mathscr{D}_{\text {eo }}$,

$$
t_{+\emptyset}^{\mathrm{o}}=\frac{z_{+} \mathscr{D}_{\mathrm{o}+}}{z_{+} \mathscr{D}_{\mathrm{o}+}-z_{-} \mathscr{D}_{\mathrm{o}-}} \quad \text { and } \quad \mathscr{D}_{\mathrm{eo}}=\frac{\left(z_{+}-z_{-}\right) \mathscr{D}_{\mathrm{o}+} \mathscr{D}_{\mathrm{o}-}}{z_{+} \mathscr{D}_{\mathrm{o}+}-z_{-} \mathscr{D}_{\mathrm{o}-}} .
$$

It is probably not possible to implement a binary oxygen/salt solution, but these definitions provide significant notational convenience.

If the cation transference number relative to solvent differs from the cation transference number relative to oxygen (i.e., if $t_{+\phi}^{0} \neq t_{+\emptyset}^{\mathrm{o}}$ ), ion fluxes induced by an electric field can exert drag forces on oxygen that differ from the drag forces the ion fluxes exert on solvent, inducing a flux of oxygen relative to solvent. These differences in the impact of migration can be understood as an electro-osmotic effect, similar to the phenomenon that drives water transport across polarized ionomer membranes in fuel cells. ${ }^{15}$ The magnitudes of $1 / \mathscr{D}_{\mathrm{eo}}$ and $1 / \mathscr{D}_{\mathrm{e} 0}$ quantify the significances of the drag forces that electrolyte exerts on oxygen and solvent, respectively.

Six macroscopic coefficients can ultimately be identified in the flux-explicit transport laws. Four are typical.

1. Thermodynamic electrolyte diffusivity:

$$
\mathscr{D}_{\mathrm{e}}=\frac{\mathscr{D}_{\mathrm{e} 0}\left(c_{0} \mathscr{D}_{\mathrm{eo}}+\nu_{\mathrm{e}} c_{\mathrm{e}} \mathscr{D}_{\mathrm{o} 0}\right)}{c_{0} \mathscr{D}_{\mathrm{eo}}+\nu_{\mathrm{e}} c_{\mathrm{e}} \mathscr{D}_{\mathrm{o} 0}+c_{\mathrm{o}} \mathscr{D}_{\mathrm{e} 0}}
$$

2. Thermodynamic oxygen diffusivity:

$$
\mathscr{D}_{\mathrm{o}}=\frac{\mathscr{D}_{\mathrm{o} 0}\left(c_{0} \mathscr{D}_{\mathrm{eo}}+c_{\mathrm{o}} \mathscr{D}_{\mathrm{e} 0}\right)}{c_{0} \mathscr{D}_{\mathrm{eo}}+v_{\mathrm{e}} c_{\mathrm{e}} \mathscr{D}_{\mathrm{o} 0}+c_{\mathrm{o}} \mathscr{D}_{\mathrm{e} 0}} .
$$

3. Cation transference number:

$$
t_{+}^{0}=\frac{\left(c_{0} \mathscr{D}_{\mathrm{e} o}+v_{\mathrm{e}} c_{\mathrm{e}} \mathscr{D}_{\mathrm{o} 0}\right) t_{+\varnothing}^{0}+c_{\mathrm{o}} \mathscr{D}_{\mathrm{e} 0} t_{+\emptyset}^{\mathrm{o}}}{c_{0} \mathscr{D}_{\mathrm{e} o}+v_{\mathrm{e}} c_{\mathrm{e}} \mathscr{D}_{\mathrm{o} 0}+c_{\mathrm{o}} \mathscr{D}_{\mathrm{e} 0}}
$$

4. Ionic conductivity:

$$
\begin{aligned}
\frac{1}{\kappa}= & \frac{1}{\kappa_{\emptyset}}+\frac{\nu_{\mathrm{e}} c_{\mathrm{o}} R T}{F^{2} z_{+}^{2} \nu_{+}^{2} c_{\mathrm{e}} c_{\mathrm{T}} \mathscr{D}_{\mathrm{eo}}} \\
& \times\left[t_{+\emptyset}^{\mathrm{o}}\left(1-t_{+\emptyset}^{\mathrm{o}}\right)+\frac{c_{0} \mathscr{D}_{\mathrm{e} 0}\left(t_{+\varnothing}^{0}-t_{+\emptyset}^{\mathrm{o}}\right)^{2}}{c_{0} \mathscr{D}_{\mathrm{eo}}+\nu_{\mathrm{e}} c_{\mathrm{e}} \mathscr{D}_{\mathrm{o} 0}+c_{\mathrm{o}} \mathscr{D}_{\mathrm{e} 0}}\right] .
\end{aligned}
$$

Two properties arise that are not typically considered.

5 Migration coefficient:

$$
\Xi=\frac{c_{\mathrm{o}} \mathscr{D}_{\mathrm{o} 0}\left(t_{+\phi}^{0}-t_{+\phi}^{\mathrm{o}}\right)}{c_{0} \mathscr{D}_{\mathrm{eo}}+v_{\mathrm{e}} c_{\mathrm{e}} \mathscr{D}_{\mathrm{o} 0}+c_{\mathrm{o}} \mathscr{D}_{\mathrm{e} 0}} .
$$

6 Cross diffusivity:

$$
\mathscr{X}_{\mathrm{eo}}=\frac{c_{\mathrm{T}} \mathscr{D}_{\mathrm{e} 0} \mathscr{D}_{\mathrm{o} 0}}{c_{0} \mathscr{D}_{\mathrm{eo}}+\nu_{\mathrm{e}} c_{\mathrm{e}} \mathscr{D}_{\mathrm{o} 0}+c_{\mathrm{o}} \mathscr{D}_{\mathrm{e} 0}} .
$$

Since this is a four-species electrolyte, it naturally affords one ionic conductivity $(\kappa)$, two dimensionless transport numbers $\left(t_{+}^{0}\right.$ and $\Xi)$, and three diffusivities $\left(\mathscr{D}_{\mathrm{e}}, \mathscr{D}_{\mathrm{o}}\right.$, and $\left.\mathscr{X}_{\mathrm{eo}}\right)$. If needed, an anion transference number can be identified by noting that $t_{0}^{0}=0$ because the solvent velocity is the reference for convection, $t_{\mathrm{o}}^{0}=0$ because both oxygen and solvent are uncharged, and $\sum_{i} t_{i}^{0}=1$ for consistency with Faraday's law, so $t_{-}^{0}=1-t_{+}^{0 .}{ }^{9}$ Equations 6 through 11 can be inverted to express the Stefan-Maxwell coefficients as explicit functions of $\kappa, t_{+}^{0}, \Xi, \mathscr{D}_{\mathrm{e}}, \mathscr{D}_{\mathrm{o}}$, and $\mathscr{X}_{\mathrm{eo}}$, as well. (These relationships are provided in the Appendix.)

\section{Transport Laws for Air-Battery Electrolytes}

Implementation of the inversion procedure and insertion of the properties defined in equations 6 through 11 result in a MacInnes 
equation involving oxygen gradients,

$$
\vec{i}=-\frac{\kappa}{F z_{+}} \vec{\nabla} \mu_{+}+\frac{\left(1-t_{+}^{0}\right) \kappa}{F z_{+} \nu_{+}} \vec{\nabla} \mu_{\mathrm{e}}+\frac{\left(z_{+}-z_{-}\right) \Xi \kappa}{F z_{+} z_{-}} \vec{\nabla} \mu_{\mathrm{o}} .
$$

To arrive at this result the isothermal, isobaric, electroneutral GibbsDuhem equation

$$
c_{0} \vec{\nabla} \mu_{0}+c_{\mathrm{o}} \vec{\nabla} \mu_{\mathrm{o}}+c_{\mathrm{e}} \vec{\nabla} \mu_{\mathrm{e}}=0
$$

has been used, in which $\mu_{\mathrm{e}}=\nu_{+} \mu_{+}+\nu_{-} \mu_{-}$. The inversion process further yields equations in Nernst-Planck form that describe the excess fluxes of oxygen, anions, and cations:

$$
\begin{gathered}
\vec{N}_{\mathrm{o}}-c_{\mathrm{o}} \vec{v}_{0}=\frac{\mathscr{X}_{\mathrm{eo}} c_{\mathrm{o}}}{c_{\mathrm{T}}} \vec{d}_{\mathrm{e}}+\mathscr{D}_{\mathrm{o}} \vec{d}_{\mathrm{o}}-\frac{\left(z_{+}-z_{-}\right) \Xi_{z_{+}}}{F z_{+}}, \\
\vec{N}_{-}-c_{-} \vec{v}_{0}=\mathscr{D}_{\mathrm{e}} \nu_{-} \vec{d}_{\mathrm{e}}+\frac{\mathscr{X}_{\mathrm{eo}} c_{-}}{c_{\mathrm{T}}} \vec{d}_{\mathrm{o}}+\frac{\left(1-t_{+}^{0}\right)}{F z_{-}} \vec{i} \\
\vec{N}_{+}-c_{+} \vec{v}_{0}=\mathscr{D}_{\mathrm{e}} \nu_{+} \vec{d}_{\mathrm{e}}+\frac{\mathscr{X}_{\mathrm{eo}} c_{+}}{c_{\mathrm{T}}} \vec{d}_{\mathrm{o}}+\frac{t_{+}^{0}}{F z_{+}} \vec{i}
\end{gathered}
$$

Here the current density $\vec{i}$, related to the fluxes through Faraday's law $\vec{i}=F \sum_{i} z_{i} \vec{N}_{i}$, is taken to drive migration; the solvent velocity $\vec{v}_{0}=\vec{N}_{0} / c_{0}$ drives convection. Two thermodynamic forces driving diffusion of the neutral-solute components, $\vec{d}_{\mathrm{e}}$ and $\vec{d}_{\mathrm{o}}$, appear:

$$
\vec{d}_{\mathrm{e}}=-\frac{c_{\mathrm{T}} c_{\mathrm{e}} \vec{\nabla} \mu_{\mathrm{e}}}{c_{0} \nu_{\mathrm{e}} R T} \quad \text { and } \quad \vec{d}_{\mathrm{o}}=-\frac{c_{\mathrm{T}} c_{\mathrm{o}} \vec{\nabla} \mu_{\mathrm{o}}}{c_{0} R T} .
$$

Note that $\vec{d}_{i}$ has been expressed with units of molar-concentration gradient here for convenience.

Under isothermal, isobaric conditions, the Gibbs phase rule requires that the component chemical potentials depend at most on $c_{\mathrm{e}}$ and $c_{\mathrm{o}}$. Thus the thermodynamic driving forces can be expressed as linear combinations of $\vec{\nabla} c_{\mathrm{e}}$ and $\vec{\nabla} c_{\mathrm{o}}$, through thermodynamic factors $\chi_{i j}\left(c_{\mathrm{e}}, c_{\mathrm{o}}\right)$,

$$
\begin{aligned}
& \vec{d}_{\mathrm{e}}=-\chi_{\mathrm{ee}} \vec{\nabla} c_{\mathrm{e}}-\chi_{\mathrm{eo}} \vec{\nabla} c_{\mathrm{o}}, \\
& \vec{d}_{\mathrm{o}}=-\chi_{\mathrm{oe}} \vec{\nabla} c_{\mathrm{e}}-\chi_{\mathrm{oo}} \vec{\nabla} c_{\mathrm{o}} .
\end{aligned}
$$

Assuming standard constitutive laws ${ }^{16,17}$

$$
\begin{aligned}
& \mu_{\mathrm{e}}\left(T, p, c_{\mathrm{e}}, c_{\mathrm{o}}\right)=\mu_{\mathrm{e}}^{\theta}(T, p)+\nu_{\mathrm{e}} R T \ln \left(f_{\mathrm{e}} c_{\mathrm{e}}\right), \\
& \mu_{\mathrm{o}}\left(T, p, c_{\mathrm{e}}, c_{\mathrm{o}}\right)=\mu_{\mathrm{o}}^{\theta}(T, p)+R T \ln \left(f_{\mathrm{o}} c_{\mathrm{o}}\right),
\end{aligned}
$$

where $\mu_{i}^{\theta}$ is the chemical potential in a primary reference state, $f_{\mathrm{e}}$ is the mean molar activity coefficient of the electrolyte, and $f_{\mathrm{o}}$ is the activity coefficient of oxygen on a molar basis, one can write generally that

$$
\chi_{i j}=\frac{c_{\mathrm{T}}}{c_{0}}\left[\delta_{i j}+c_{i}\left(\frac{\partial \ln f_{i}}{\partial c_{j}}\right)_{T, p, c_{k \neq j}}\right],
$$

in which $\delta_{i j}$ is the Kronecker delta. For an ideal neutral component $i, f_{i}$ is independent of composition; in the limit of infinite dilution, $c_{\mathrm{T}} \approx c_{0}$. Thus the force driving mass transport of neutral-solute component $i$ in an ideal, dilute air-battery electrolyte is $\vec{d}_{i} \approx-\vec{\nabla} c_{i}$.

\section{Consequences of Ion/Oxygen Interactions}

Existing models of air-battery electrolytes tend to use the equations from Nernst-Planck theory or the theory of concentrated binary electrolytes to describe the ion fluxes, and additionally adopt Fick's law to describe the oxygen flux. This theoretical structure is (uniquely) achieved by taking $\Xi$ and $\mathscr{X}_{\text {eo }}$ to vanish in Equations 12 and 14-16, leaving a theory with four transport coefficients.

Given a system in which the species concentrations are all finite, the conditions $\mathscr{X}_{\mathrm{eo}} \rightarrow 0$ and $\Xi \rightarrow 0$ imply through Equations A3 and A4 (in the Appendix) that $1 / \mathscr{D}_{\mathrm{o}+} \rightarrow 0$ and $1 / \mathscr{D}_{\mathrm{o}-} \rightarrow 0$; conversely, the conditions $1 / \mathscr{D}_{\mathrm{o}+} \rightarrow 0$ and $1 / \mathscr{D}_{\mathrm{o}-} \rightarrow 0$ imply through equations
5,10 , and 11 that $\Xi \rightarrow 0$ and $\mathscr{X}_{\mathrm{eo}} \rightarrow 0$. Thus the migration coefficient and cross-diffusivity vanish if and only if the Stefan-Maxwell diffusional resistances associated with microscopic ion/oxygen interactions vanish. Prior models therefore result from neglecting drag forces that ions could exert on oxygen.

In all known air-battery electrolytes the maximum oxygen concentration is extremely small in comparison to the concentrations of both salt and solvent, ${ }^{18}$ which is a limit worth exploring. In this case one is not justified in neglecting the cross-diffusion terms in Equations 15 and 16; although $\left\|\vec{d}_{\mathrm{o}}\right\| \sim\left\|\vec{\nabla} c_{\mathrm{o}}\right\|$, the fact that $c_{\mathrm{o}} \ll c_{\mathrm{e}}$ does not require that $\left\|\vec{\nabla} c_{\mathrm{o}}\right\| \ll\left\|\vec{\nabla} c_{\mathrm{e}}\right\|$. (Indeed, at the beginning of oxygen exposure under open-circuit conditions, these two gradients are of the same order of magnitude.) Since $\Xi \propto c_{\mathrm{o}}$, the molar migration coefficient $\tilde{\Xi}$, defined as

$$
\tilde{\Xi}=\frac{\Xi c_{\mathrm{T}}}{c_{\mathrm{o}}},
$$

remains finite as $c_{\mathrm{o}} \rightarrow 0$ if the Stefan-Maxwell diffusivities are all finite. Incorporation of $\tilde{\Xi}$ into the oxygen flux law (Equation 14) shows that the $\vec{d}_{\mathrm{e}}, \vec{d}_{\mathrm{o}}$, and $\vec{i}$ terms are all of $O\left(c_{\mathrm{o}}\right)$; no term can be neglected on the grounds that $c_{\mathrm{o}}$ is small.

It would be thermodynamically inconsistent to incorporate a concentration overpotential associated with oxygen gradients (such as the $\vec{\nabla} \mu_{\mathrm{o}}$ term in Equation 12) without placing a corresponding migration term in the flux law for oxygen (such as the $\vec{i}$ term in Equation 14). Significant modification of all the transport laws may be needed to account for effects of the oxygen distribution on cell potential.

Assuming a reference electrode reversible only to cations is used to measure the voltage drop across the cell interior (so that $\vec{\nabla} \mu_{+}=$ $\left.F z_{+} \vec{\nabla} \Phi\right)$, equation 12 yields a law that governs the change in electrode potential $\Phi$,

$$
-\vec{\nabla} \Phi=\frac{\vec{i}}{\kappa}+\frac{\nu_{\mathrm{e}} R T c_{0}}{F z_{+} \nu_{+} c_{\mathrm{T}} c_{\mathrm{e}}}\left[\left(1-t_{+}^{0}\right) \vec{d}_{\mathrm{e}}-\tilde{\Xi} \frac{c_{\mathrm{e}}}{c_{\mathrm{T}}} \vec{d}_{\mathrm{o}}\right] .
$$

The term with $\vec{d}_{\mathrm{o}}$ scales as $-\vec{\nabla} c_{\mathrm{o}}$. Thus large oxygen gradients can induce an electric field-even if $c_{\mathrm{o}}$ is very low.

\section{Oxygen Diffusion Potential During Lithium/Oxygen Preconditioning}

Equation 22 rationalizes the voltage response observed during a standard preconditioning experiment - formally, an open-circuit hold of a planar air-battery cell initially equilibrated under argon, whose ambient gas undergoes a sudden change from argon to fixed-pressure oxygen at time $t=0$. Immediately after the cell is exposed to oxygen (time $t=0^{+}$), the oxygen concentration in the liquid at the gas interface, $c_{\mathrm{o}}^{\mathrm{pos}}$, will rapidly reach the saturated liquid-phase oxygen concentration $c_{\mathrm{o}}^{\text {sat }}$ if the kinetics of oxygen absorption into the liquid is fast in comparison to oxygen diffusion rates. During this very early time, the cell potential will rise quite rapidly from the equilibrium potential achieved at open circuit under argon to a value somewhat above or below the equilibrium potential under oxygen.

At sufficiently short times after the kinetic relaxation associated with oxygen sorption, diffusing oxygen will have reached its equilibrium concentration within the liquid at the gas interface, but will not have penetrated through the separator, so the oxygen concentration at the anode/separator interface, $c_{\mathrm{o}}^{\text {neg }}$, will not equal $c_{\mathrm{o}}^{\text {sat }}$. Whenever such a difference exists within the cell, a concentration overpotential arising from oxygen may affect the cell voltage. If present, this overpotential would be expected to relax on a diffusional timescale, which - unlike the kinetic relaxation - would depend on the cell thickness. The discussion that follows analyzes the diffusional relaxations of oxygen and salt during preconditioning, with the aim of determining whether oxygen migration is significant in lithium/oxygen cells.

Liu et al. ${ }^{2}$ listed a set of thermodynamic parameters that describe $1 \mathrm{M}$ LiTFSI in dimethoxyethane - a standard electrolyte for lithium/oxygen batteries. At room temperature the saturated 
oxygen concentration in this electrolyte is $c_{\mathrm{o}}^{\text {sat }}=0.0021 \mathrm{M}$. The partial molar volumes of solvent and salt are $\bar{V}_{0}=0.1043 \mathrm{M}^{-1}$ and $\bar{V}_{\mathrm{e}}=0.0210 \mathrm{M}^{-1}$, respectively. With these parameters one finds in the absence of oxygen that $c_{0}=9.4 \mathrm{M}$ and $c_{\mathrm{T}}=11.4 \mathrm{M}$, and that the volume fraction occupied by solvent is $c_{0} \bar{V}_{0}=0.98$. An assumption that saturation with oxygen negligibly affects liquid volume implies that $\bar{V}_{\mathrm{o}} \rightarrow 0$. These values confirm that $c_{0}$ and $c_{\mathrm{e}}$ are both of the order of $c_{\mathrm{T}}$, whereas $c_{\mathrm{o}} \ll c_{\mathrm{T}}$.

The assumption that $c_{\mathrm{o}} \ll c_{\mathrm{T}}$ simplifies the transport properties substantially. As one might expect, Equations 6, 8, and 9 show in the limit that the electrolyte diffusivity, cation transference number, and ionic conductivity match their values in the absence of oxygen,

$$
\lim _{c_{\mathrm{o}} \ll c_{\mathrm{T}}} \mathscr{D}_{\mathrm{e}}=\mathscr{D}_{\mathrm{e} 0}, \lim _{c_{\mathrm{o}} \ll c_{\mathrm{T}}} t_{+}^{0}=t_{+\phi}^{0}, \text { and } \lim _{c_{\mathrm{o}} \ll c_{\mathrm{T}}} \kappa=\kappa_{\phi} .
$$

Interestingly, the oxygen diffusivity in this limit differs substantially from $\mathscr{D}_{\mathrm{o} 0}$ :

$$
\lim _{c_{\mathrm{o}} \ll c_{\mathrm{T}}} \frac{1}{\mathscr{D}_{\mathrm{o}}}=\frac{1}{\mathscr{D}_{\mathrm{o} 0}}+\frac{\nu_{\mathrm{e}} c_{\mathrm{e}}}{c_{0} \mathscr{D}_{\mathrm{eo}}} .
$$

Thus one straightforward way to evaluate the strength of the diffusional resistance that electrolyte and oxygen exert on each other, $1 / \mathscr{D}_{\mathrm{eo}}$, would be to measure how much the apparent oxygen diffusivity reduces upon the addition of salt to the pure solvent.

Liu et al. also presented Stefan-Maxwell diffusivities for $1 \mathrm{M}$ LiTFSI in dimethoxyethane that yield $\mathscr{D}_{\mathrm{e}}=5.65 \times 10^{-10} \mathrm{~m}^{2} \mathrm{~s}^{-1}$, $t_{+}^{0}=0.43$, and $\kappa=36.9 \mathrm{mScm}^{-1} ;^{2} \mathrm{Lu}$ et al. report for the same electrolyte that $\mathscr{D}_{\mathrm{o}}=7.3 \times 10^{-10} \mathrm{~m}^{2} \mathrm{~s}^{-1} \cdot{ }^{19}$ The values of $\tilde{\Xi}$ and $\mathscr{X}_{\mathrm{eo}}$ depend primarily on oxygen/salt interactions and remain unknown.

Given the low saturated concentration of oxygen, it is reasonable to treat it as a thermodynamically ideal solute, so that $f_{\mathrm{o}}=1$, implying that $\chi_{\mathrm{oe}}=0$ and $\chi_{\mathrm{oo}}=c_{\mathrm{T}} / c_{0}$. For simplicity we assume $\chi_{\mathrm{eo}}=0$ as well (i.e., salt activity is unaffected by oxygen content), which allows $\chi_{\mathrm{ee}}$ to be estimated from properties of oxygen-free electrolytes. Generally $\chi_{\text {ee }}$ is of order unity; typical values are $c_{0} \bar{V}_{0} \chi_{\text {ee }}=0.68$ for $2.24 \mathrm{M} \mathrm{LiPF}_{6}$ in a mixed-carbonate solvent and $c_{0} \bar{V}_{0} \chi_{\mathrm{ee}}=3.1$ for $0.85 \mathrm{M} \mathrm{LiPF}_{6}$ in propylene carbonate. ${ }^{20}$ For the purposes of the present analysis we take $\chi_{\text {ee }}=5$ (independent of composition), an atypically large value that should amplify the concentration overpotential contributed by salt gradients.

Finally, it is reasonable to assume that the variations in $c_{0}$ and $c_{\mathrm{e}}$ are of similar order as the variations of $c_{\mathrm{o}}$ during a preconditioning experiment. Thus oxygen transport can be thought of as inducing a first-order regular perturbation to the system that governs transport in oxygen's absence, while the transport properties, thermodynamic factors, and partial molar volumes can be assumed constant and equal to their values in the limit $c_{\mathrm{o}} \rightarrow 0$.

The transient cell response is governed by four species material balances of the form

$$
\frac{\partial c_{i}}{\partial t}=-\vec{\nabla} \cdot \vec{N}_{i}
$$

Under the justifiable assumption that the electrolyte maintains local electroneutrality, two of these balances can be replaced with equations that respectively express the local continuity of charge and volume, ${ }^{21}$

$$
\vec{\nabla} \cdot \vec{i}=0 \quad \text { and } \quad \vec{\nabla} \cdot \vec{v}^{\square}=0 .
$$

Here $\vec{v}^{\square}$ represents the volume-average velocity of the liquid electrolyte, $\vec{v} \square=\sum_{i} \bar{V}_{i} \vec{N}_{i}$. The planar cell is held at open circuit during a preconditioning period, so the charge balance implies that current density vanishes uniformly, $\vec{i}=\overrightarrow{0}$. Also, no species cross the anode during an open-circuit hold, so $\vec{v} \square$ must vanish there, whence volume continuity implies $\vec{v}^{\square}=\overrightarrow{0}$ uniformly as well.

The flux laws for oxygen and lithium cations during a preconditioning interval in a planar cell containing an electrolytic solution like
1 M LiTFSI in dimethoxyethane therefore simplify to

$$
\begin{gathered}
\vec{N}_{\mathrm{o}}=-\frac{\left\langle c_{\mathrm{T}}\right\rangle \mathscr{D}_{\mathrm{o}}}{\left\langle c_{0}\right\rangle} \vec{\nabla} c_{\mathrm{o}} \\
\vec{N}_{+}=-\nu_{+}\left\langle c_{\mathrm{T}}\right\rangle \bar{V}_{0}\left(\mathscr{D}_{\mathrm{e}} \chi_{\mathrm{ee}} \vec{\nabla} c_{\mathrm{e}}+\frac{\left\langle c_{\mathrm{e}}\right\rangle \mathscr{X}_{\mathrm{eo}}}{\left\langle c_{\mathrm{T}}\right\rangle} \vec{\nabla} c_{\mathrm{o}}\right) .
\end{gathered}
$$

(Angled brackets around a quantity indicate the value in an oxygenfree equilibrated state.) Oxygen therefore does obey a form of Fick's law in the special case at hand, but only because the open-circuit condition removes any contribution that migration might make to the oxygen flux. Despite the null applied current, oxygen can still contribute to the diffusion potential; the MacInnes equation during preconditioning becomes

$$
\frac{F z_{+} \nu_{+}\left\langle c_{\mathrm{T}}\right\rangle}{\nu_{\mathrm{e}} R T} \vec{\nabla} \Phi=\frac{\chi_{\mathrm{ee}}\left(1-t_{+}^{0}\right)\left\langle c_{0}\right\rangle}{\left\langle c_{\mathrm{e}}\right\rangle} \vec{\nabla} c_{\mathrm{e}}-\tilde{\Xi} \vec{\nabla} c_{\mathrm{o}},
$$

in which the second term on the right may be substantially larger in magnitude than the first.

Insertion of Equation 27 into Equation 25 produces the familiar diffusion equation

$$
\frac{\partial c_{\mathrm{o}}}{\partial t}=\frac{\left\langle c_{\mathrm{T}}\right\rangle \mathscr{D}_{\mathrm{o}}}{\left\langle c_{0}\right\rangle} \frac{\partial^{2} c_{\mathrm{o}}}{\partial x^{2}},
$$

whereas inserting Equation 28 into Equation 25 yields a more unusual balance for the dissolved electrolyte,

$$
\frac{1}{\left\langle c_{\mathrm{T}}\right\rangle \bar{V}_{0}} \frac{\partial c_{\mathrm{e}}}{\partial t}=\mathscr{D}_{\mathrm{e}} \chi_{\mathrm{ee}} \frac{\partial^{2} c_{\mathrm{e}}}{\partial x^{2}}+\frac{\left\langle c_{\mathrm{e}}\right\rangle \mathscr{X}_{\mathrm{eo}}}{\left\langle c_{\mathrm{T}}\right\rangle} \frac{\partial^{2} c_{\mathrm{o}}}{\partial x^{2}} .
$$

Here $x$ denotes the position normal to the negative electrode (anode), assumed to be a slab of lithium metal.

A standard preconditioning experiment begins with initial conditions

$$
c_{\mathrm{o}}(0, x)=0 \quad \text { and } \quad c_{\mathrm{e}}(0, x)=\left\langle c_{\mathrm{e}}\right\rangle .
$$

The boundary conditions during the open-circuit hold after oxygen exposure are

$$
c_{\mathrm{o}}(t, L)=c_{\mathrm{o}}^{\mathrm{sat}}, N_{\mathrm{o}}(t, 0)=0, \text { and } N_{+}(t, 0)=0,
$$

where $x=L$ corresponds to the position of the liquid/gas interface. Since lithium ions are neither produced nor consumed within the resting cell, one can also write an auxiliary condition

$$
\frac{1}{L} \int_{0}^{L} c_{\mathrm{e}}(t, x) d x=\left\langle c_{\mathrm{e}}\right\rangle,
$$

which holds throughout the preconditioning period.

Equations 30 through 34 comprise a well-posed description of mass transport during preconditioning. The transient oxygen and electrolyte distributions yielded by solving this governing system can then be inserted into Equation 29 to evaluate the voltage relaxation that accompanies oxygen absorption into the liquid electrolyte.

Solving the problem is more straightforward than it appears because the transient oxygen balance is decoupled from the salt balance. The difference in oxygen concentration across the cell is found to vary with time after exposure to pure oxygen gas as

$$
\begin{aligned}
\Delta c_{\mathrm{o}}(t) & =c_{\mathrm{o}}^{\mathrm{pos}}(t)-c_{\mathrm{o}}^{\mathrm{neg}}(t)=c_{\mathrm{o}}(t, L)-c_{\mathrm{o}}(t, 0) \\
& =-\frac{4 c_{\mathrm{o}}^{\mathrm{sat}}}{\pi} \sum_{n=1}^{\infty} \frac{(-1)^{n}}{2 n-1} \exp \left[-\frac{\pi^{2}(2 n-1)^{2}\left\langle c_{\mathrm{T}}\right\rangle \mathscr{D}_{\mathrm{o}} t}{4\left\langle c_{0}\right\rangle L^{2}}\right] .
\end{aligned}
$$

This is obtained by solving governing Equation 30 subject to the first of initial conditions 32 , as well as the boundary conditions on $c_{\mathrm{o}}$ and $N_{\mathrm{o}}$, none of which involves $c_{\mathrm{e}}(t, x)$.

With equation 35 in hand it is possible to solve coupled governing Equation 31, subject to the second of initial conditions 32, the boundary condition on $N_{+}$, and auxiliary condition 34 . This process 
is facilitated by Laplace transformation with respect to time, which yields

$$
\begin{aligned}
& \Delta c_{\mathrm{e}}(t)=\frac{\bar{V}_{0}\left\langle c_{0}\right\rangle \mathscr{X}_{\mathrm{eo}}\left\langle c_{\mathrm{e}}\right\rangle c_{\mathrm{o}}^{\mathrm{sat}}}{\left(\mathscr{D}_{\mathrm{o}}-\bar{V}_{0}\left\langle c_{0}\right\rangle \mathscr{D}_{\mathrm{e}} \chi_{\mathrm{ee}}\right)\left\langle c_{\mathrm{T}}\right\rangle}\left\{\frac{\Delta c_{\mathrm{o}}(t)}{c_{\mathrm{o}}^{\mathrm{sat}}}+\frac{32 \mathscr{D}_{\mathrm{o}}}{\pi^{2}}\right. \\
& \times \sum_{m=1}^{\infty} \sum_{n=1}^{\infty} \frac{\exp \left[-\frac{\pi^{2}(2 n-1)^{2}\left\langle c_{\mathrm{T}}\right\rangle \mathscr{D}_{\mathrm{o}} t}{4\left\langle c_{0}\right\rangle L^{2}}\right]}{\mathscr{D}_{\mathrm{o}}(2 n-1)^{2}-4 \bar{V}_{0}\left\langle c_{0}\right\rangle \mathscr{D}_{\mathrm{e}} \chi_{\mathrm{ee}}(2 m-1)^{2}}-\frac{32 \mathscr{D}_{\mathrm{o}}}{\pi^{2}} \\
& \left.\times \sum_{m=1}^{\infty} \sum_{n=1}^{\infty} \frac{\exp \left[-\frac{\pi^{2}(2 m-1)^{2}\left\langle c_{\mathrm{T}}\right\rangle \mathscr{D}_{\mathrm{e}} \chi_{\mathrm{ee}} t}{4 L^{2}}\right]}{\mathscr{D}_{\mathrm{o}}(2 n-1)^{2}-4 \bar{V}_{0}\left\langle c_{0}\right\rangle \mathscr{D}_{\mathrm{e}} \chi_{\mathrm{ee}}(2 m-1)^{2}}\right\}
\end{aligned}
$$

for the time evolution of the difference in salt concentration across the separator.

The prefactor on the right of Equation 36 involves the single unknown parameter $\mathscr{X}_{\mathrm{eo}}$. Although the cross-diffusivity is not known precisely, physical reasoning places bounds on it:

$$
0 \leq \mathscr{X}_{\mathrm{eo}}<\frac{\mathscr{D}_{\mathrm{e}}\left\langle c_{\mathrm{T}}\right\rangle}{\nu_{\mathrm{e}}\left\langle c_{\mathrm{e}}\right\rangle} .
$$

Equations 27 and 28 involve a transport matrix that stability requires to be positive definite, implying that $\mathscr{D}_{\mathrm{o}}>0$. Through Equations 11 and 24 , this inequality places the upper bound on $\mathscr{X}_{\text {eo. }}$. The lower bound arises from inspection of Equation 24, which shows that $\mathscr{D}_{\text {eo }}$ leads to increased diffusional drag on oxygen by the electrolyte. Salt exerts the least additional drag in the limit $\mathscr{D}_{\text {eo }} \rightarrow \infty$, a condition that equation 11 shows to correspond with $\mathscr{X}_{\mathrm{eo}} \rightarrow 0$.

Integration of Equation 29 demonstrates that the potential drop across the separator during preconditioning follows

$$
\frac{F z_{+} \nu_{+}}{\nu_{\mathrm{e}} R T} \Delta \Phi=\frac{\left\langle c_{0}\right\rangle \chi_{\mathrm{ee}}\left(1-t_{+}^{0}\right)}{\left\langle c_{\mathrm{T}}\right\rangle\left\langle c_{\mathrm{e}}\right\rangle} \Delta c_{\mathrm{e}}-\frac{\tilde{\Xi}}{\left\langle c_{\mathrm{T}}\right\rangle} \Delta c_{\mathrm{o}} .
$$

The greatest potential drop occurs at $t=0$. Experiments using lithium perchlorate in diglyme - an ethereal electrolyte similar to LiTFSI in dimethoxyethane - suggest that $\Delta \Phi(0)$ is of the order of $-100 \mathrm{mV} .^{22}$ Inserting all of the property values listed in this section, and assuming the maximum possible value for $\mathscr{X}_{\mathrm{eo}}$, one finds that the $\Delta c_{\mathrm{e}}$ term in Equation 38 is more than three orders of magnitude smaller than the $\Delta \Phi$ term. The potential drop can be explained by the $\Delta c_{0}$ term if $\tilde{\Xi} \approx 1.1 \times 10^{4}$; the corresponding migration coefficient is $\Xi=1.9$. On the basis of this estimate, we hypothesize that the electro-osmotic effect could be significant in lithium/oxygen cells, and encourage its consideration in air-battery modeling and characterization. It is worth noting that the prefactor of the current density in Equation 14 is of the same order as those in Equations 15 and 16. Thus the fluxes of oxygen, cations, and anions due to migration all appear to have similar magnitudes.

Liquid electrolytes with minimal ion/oxygen drag interactions will exhibit apparently lower overpotentials when operated at higher rates, a range where oxygen concentration polarization across the cell is expected to be significant. Targeting electrolytes with low migration coefficients could be a route by which to improve the energy efficiencies of air-battery systems.

\section{Acknowledgment}

This work was supported by the US National Science Foundation, grant no. CBET 1336387.

\section{Appendix}

The five Stefan-Maxwell coefficients $\mathscr{D}_{0+}, \mathscr{D}_{0-}, \mathscr{D}_{0^{+}}, \mathscr{D}_{0^{-}}$, and $\mathscr{D}_{00}$ are expressed in terms of the five macroscopic properties $\mathscr{D}_{\mathrm{e}}, \mathscr{D}_{\mathrm{o}}, \mathscr{X}_{\mathrm{e} 0}, t_{+}^{0}$, and $\Xi$ as

$$
\begin{gathered}
\mathscr{D}_{0+}=\frac{\frac{v_{+}}{v_{\mathrm{e}}}\left(\mathscr{D}_{\mathrm{e}} \mathscr{D}_{\mathrm{o}}-\frac{v_{\mathrm{e}} c_{\mathrm{c}} c_{\mathrm{o}}}{c_{\mathrm{T}}^{2}} \mathscr{X}_{\mathrm{eo}}^{2}\right)}{\left(\mathscr{D}_{\mathrm{o}}-\frac{c_{\mathrm{o}}}{c_{\mathrm{T}}} \mathscr{X}_{\mathrm{eo}}\right)\left(1-t_{+}^{0}\right)-\left(\mathscr{D}_{\mathrm{e}}-\frac{v_{\mathrm{e}} c_{\mathrm{e}}}{c_{\mathrm{T}}} \mathscr{X}_{\mathrm{eo}}\right) \Xi}, \\
\mathscr{D}_{0-}=\frac{\frac{v_{-}}{v_{\mathrm{e}}}\left(\mathscr{D}_{\mathrm{e}} \mathscr{D}_{\mathrm{o}}-\frac{v_{\mathrm{e}} c_{e} c_{\mathrm{o}}}{c_{\mathrm{T}}^{2}} \mathscr{X}_{\mathrm{eo}}^{2}\right)}{\left(\mathscr{D}_{\mathrm{o}}-\frac{c_{\mathrm{o}}}{c_{\mathrm{T}}} \mathscr{X}_{\mathrm{eo}}\right) t_{+}^{0}+\left(\mathscr{D}_{\mathrm{e}}-\frac{v_{\mathrm{e}} c_{\mathrm{e}}}{c_{\mathrm{T}}} \mathscr{X}_{\mathrm{eo}}\right) \Xi}, \\
\mathscr{D}_{\mathrm{o}+}=\frac{\frac{v_{+} c_{\mathrm{o}}}{v_{\mathrm{e}} c_{0}}\left(\mathscr{D}_{\mathrm{e}} \mathscr{D}_{\mathrm{o}}-\frac{v_{\mathrm{e}} c_{\mathrm{e}} c_{\mathrm{o}}}{c_{\mathrm{T}}^{2}} \mathscr{X}_{\mathrm{eo}}^{2}\right)}{\frac{c_{\mathrm{o}}}{c_{\mathrm{T}}} \mathscr{X}_{\mathrm{eo}}\left(1-t_{+}^{0}\right)+\mathscr{D}_{\mathrm{e}} \Xi}, \\
\mathscr{D}_{\mathrm{o}-}=\frac{\frac{v_{-} c_{\mathrm{o}}}{v_{\mathrm{e}} c_{0}}\left(\mathscr{D}_{\mathrm{e}} \mathscr{D}_{\mathrm{o}}-\frac{v_{\mathrm{e}} c_{\mathrm{c}} c_{\mathrm{o}}}{c_{\mathrm{T}}^{2}} \mathscr{X}_{\mathrm{eo}}^{2}\right)}{\frac{c_{\mathrm{o}}}{c_{\mathrm{T}}} \mathscr{X}_{\mathrm{e}} t_{+}^{0}-\mathscr{D}_{\mathrm{e}} \Xi}, \\
\mathscr{D}_{\mathrm{o} 0}=\frac{\mathscr{D}_{\mathrm{e}} \mathscr{D}_{\mathrm{o}}-\frac{v_{\mathrm{e}} c_{\mathrm{e}} c_{\mathrm{o}}}{c_{\mathrm{T}}^{2}} \mathscr{X}_{\mathrm{eo}}^{2}}{\mathscr{D}_{\mathrm{e}}-\frac{v_{\mathrm{e}} c_{\mathrm{e}}}{c_{\mathrm{T}}} \mathscr{X}_{\mathrm{eo}}} .
\end{gathered}
$$

Given these relationships, one can compute $\mathscr{D}_{+-}$as a function of $\kappa, \mathscr{D}_{\mathrm{e}}, \mathscr{D}_{\mathrm{o}}, \mathscr{X}_{\mathrm{e}}, t_{+}^{0}$, and $\Xi$ through

$$
\begin{aligned}
\frac{1}{\mathscr{D}_{+-}}= & -\frac{F^{2} c_{\mathrm{T}} z_{+} z_{-}}{R T \kappa}+\frac{v_{\mathrm{e}} c_{0}\left(t_{+\emptyset}^{\mathrm{o}}-t_{+\emptyset}^{0}\right) \Xi}{v_{+} \nu_{-} c_{\mathrm{e}} \mathscr{D}_{0} 0} \\
& -\frac{1}{c_{\mathrm{e}}}\left(\frac{c_{0}}{v_{-} \mathscr{D}_{0+}+v_{+} \mathscr{D}_{0-}}+\frac{c_{\mathrm{o}}}{v_{-} \mathscr{D}_{0+}+v_{+} \mathscr{D}_{0-}}\right) .
\end{aligned}
$$

\section{References}

1. L. Onsager, Annals of the New York Academy of Sciences, 46, 241 (1945).

2. J. Liu, S. Khaleghi Rahimian, and C. W. Monroe, Physical Chemistry Chemical Physics, 18, 22840 (2016).

3. P. Albertus, G. Girishkumar, B. McCloskey, R. Sánchez-Carrera, B. Kozinsky, J. Christensen, and A. Luntz, Journal of the Electrochemical Society, 158, A343 (2011).

4. P. Andrei, J. Zheng, M. Hendrickson, and E. Plichta, Journal of the Electrochemical Society, 159, A770 (2012).

5. B. Horstmann, T. Danner, and W. Bessler, Energy \& Environmental Science, 6, 1299 (2013).

6. U. Sahapatsombut, H. Cheng, and K. Scott, Journal of Power Sources, 227, 243 (2013).

7. K. Yoo, S. Banerjee, and P. Dutta, Journal of Power Sources, 258, 340 (2014)

8. J. Newman and K. E. Thomas-Alyea, Electrochemical Systems, $3^{\text {rd }}$ Edition John Wiley \& Sons, Inc.: Hoboken, New Jersey, 2004

9. C. W. Monroe and C. Delacourt, Electrochimica Acta, 114, 649 (2013).

10. D. A. MacInnes, The Principles of Electrochemistry, $2^{\text {nd }}$ Ed. Dover Publications, Inc.: New York, New York, 1961.

11. Y.-C. Lu, H. Gasteiger, M. Parent, V. Chiloyan, and Y. Shao-Horn, Electrochemical and Solid-State Letters, 13, A69 (2010).

12. B. McCloskey, D. Bethune, R. Shelby, G. Girishkumar, and A. Luntz, The Journal of Physical Chemistry Letters, 2, 1161 (2011).

13. B. Adams, C. Radtke, R. Black, M. Trudeau, K. Zaghib, and L. Nazar, Energy \& Environmental Science, 6, 1772 (2013).

14. H. Geaney, J. O'Connell, J. Holmes, and C. O'Dwyer, Journal of the Electrochemical Society, 161, A1964 (2014).

15. T. F. Fuller and J. Newman, Journal of the Electrochemical Society, 139, 1332 (1992)

16. E. A. Guggenheim, Thermodynamics: an Advanced Treatment for Chemists and Physicists, $5^{\text {th }}$ Ed. North-Holland Publishing Company: Amsterdam, 1967.

17. R. A. Robinson and R. H. Stokes, Electrolyte Solutions, $2^{\text {nd }}$ Ed. (Revised) Butterworths: London, U.K., 1968.

18. J. Read, Journal of the Electrochemical Society, 153, A96 (2006)

19. Y.-C. Lu, D. G. Kwabi, K. P. C. Yao, J. R. Harding, J. Zhou, L. Zuin, and Y. Shao-Horn, Energy and Environmental Science, 4, 2999 (2011).

20. J. Liu and C. W. Monroe, Electrochimica Acta, 167, 357 (2015).

21. J. Liu and C. W. Monroe, Electrochimica Acta, 135, 447 (2014).

22. Personal communication of data gathered by Xiangwen Gao, 22 January 2016. From Lee Johnson, Peter Bruce Group, Department of Materials, University of Oxford. 